Ann. Zootech., I968, 17 (3), 289-305.

\title{
ÉTUDE DE QUELQUES FACTEURS DE LA STABILITÉ DES ANTIBIOTIQUES DANS LES ALIMENTS POUR PORCELETS
}

\author{
E. SALMON-LEGAGNELR, A. AIMAITRE, C. JOUANDET \\ arer lat collaboration technique de P. Vaissade, Inguette Dewulf, A. Kvotsant, \\ Hélène Moutel, Michelle Nocart et J. Hautducieur. \\ Station de Recherches sur l'Élerage des Porcs, \\ Centre national de Recherches zootechniques, 78 -Jouy-en-Josas \\ Institut national de la Recherche agronomique
}

\author{
SOMMAIRE
}

Le taux de disparition de l'activité antibiotique (dosage microbiologique) est très variable mais il peut dépasser 80 p. 100 avant 6 mois dans certains aliments. Cette dégradation dépend en particulier de la nature de l'antibioticue, mais aussi de la composition de l'aliment (présence de graisse) et de sa présentation (granulation) : la teneur en protides et la dose d'antibiotique semblent avoir une moindre infl tence.

\section{IN'TRODUC'TION}

Il arrive parfois que des supplémentations par un même antibiotique d'aliments destinés aux animaux ne produisent pas le même effet dans le temps. L'intérêt de catte stimulation pour favoriser la croissance s'en trouve donc diminué. I)iverses explications ont été données à ce phénomène. Enn particulier, la variabilité peut provenir des différcnces de sensibilité individuelle des animaux, des phénomènes d'accoutumance ou des rariations des conditions de milieu. Une autre explication très simple de ce manque de fidélité poutrait être la perte d'activité de l'antibiotique dans l'aliment. Cette dégradation est un phénomène connu qui a été signalé pour la plupart des antibiotiques utilisés en alimentation animale (EsPosito et al., r952; HoLLENBECK, I954; Weixstein, I958; GoldberG, I959; KATZ et FAssbender, I967; Barbiers et Neff, I966; Worxick, I967; HusaAs, I967). Nous l'avons rencontrée au cours d'une expérience sur porcelets, oì elle fut la cause probable d'une réduction importante de la stimulation de croissance escomptée (JouANDFT et al., I964). Tou- 
tefois, les facteurs qui provoquent cette dégradation au cours du stociage des aliments sont encore mal connus (WORNICK, I967).

Il nous a donc paru utile d'examiner dans le cas des aliments pour sevrage des porcelets, où l'antibiosupplémentation est souvent la règle, l'influence de quelques facteurs qui varient souvent dans les fabrications : taux de protides, présence de graisse, présentation, dose d'antibiotique.

Cette expérience a consisté à étudier l'évolution, de l'activité antibiotique, mosurée par dosage microbiologique, au couts de la conservation, de divers aliments représentant 48 combinaisons différentes ( 2 taux de protides $\times 2$ taux de lipides $\times 2$ présentations $\times 2$ doses d'antibiotique $\times 3$ antibiotiques). I a durée de stockage était fixée à 6 mois par similitude avec la garantie exigée dans les aliments industriels pour certaines vitamines.

\section{MATÉRIEL, ETT MÉTHODES}

\section{a) Antibiotiques}

Les 3 antibiotiques utilisés ont été choisis parmi ceux d'un emploi courant en alimentation animale. La forme chimique et la présentation étaient celles des préparations commerciales usuelles à l'usage de l'alimentation ( feed grade), à savoir :

- chlortétracycline base $\left({ }^{1}\right)$ : adjuvant A 80/80, Rhône-Poulenc (8I,5 g d'activité chlorhydrate $/ \mathrm{kg}$ ).

- oxytétracycline complexée sur ammonium quaternaire : terramycine $\mathrm{T}$ 8o, Pfizer-Clin (82, i g d'activité chlorhydrate $/ \mathrm{kg}$ ).

- Embonate de spiramycine : Spiravit 20, Rhône-Poulenc (22 g 75 d'activité embonate $/ \mathrm{kg}$ ).

Chacune de ces préparations était introduite sur un support de tourteau de soja cuit, compte tenu de son activité réelle, de façon à réaliser des concentrations de ro et 100 p.p.m. dans les aliments.

\section{b) Aliments}

Quatre formules d'aliments pour porcelets correspondant aux combinaisons factorielles de 2 taux de protéines ( 16 et 25 p. 100) et de 2 taux de lipides (o et ro p. Ioo d'huile stabilisée au BHT.) ont été utilisées. Les formules désignées par les lettres A, B, C, D sont reproduites dans le tableau I. A l'exception du taux de graisse volontairement élevé dans les aliments $\mathrm{A}$ et $\mathrm{C}$, la composition de ces aliments est voisine de celle que l'on trouve dans les aliments pour sevrage des porcelets de 3 à 5 semaines (starter porcelet). L'huile d'arachide, bien que peu utilisée habituellement, a été choisie de préférence à d'autres graisses pour la meilleure homogénéité qu'elle conférait aux mélanges. Chacun de ces aliments était préparé sous deux présentations différentes, farine (mouture moyenne) ou granulés $(2,5 \mathrm{~mm}$ de diamètre) par passage de la farine à travers une presse Templewood.

De façon à réduire les erreurs ou variations liées à l'hétérogénéité des aliments, la préparation de la quantité nécessaire de chaque aliment $\mathrm{A}, \mathrm{B}, \mathrm{C}$ ou $\mathrm{D}$ pour tous les antibiotiques était effectuée en une seule fois (mélangeuse de I $500 \mathrm{l}$ ). L'introduction des antibiotiques était alors effectuée aux doses voulues (mélangeuse de $200 \mathrm{l}$ ). La moitié de chaque préparation était ensuite granulée et l'autre laissée en farine. Les aliments étaient conservés dans des sacs en papier. Le stockage avait lieu dans un hangar fermé, à proximité des bâtiments d'élevage des animaux et à température ambiante. Les températures et le degré hygrométrique du local étaient enregistrés pendant toute la durée de l'expérience (tabl. 2).

\section{c) Analyses}

Toutes les analyses ont été effectuées par voie microbiologique. L'activité antibiotique des échantillons d'aliment était déterminée par rapport à une gamme de concentration connue et à des aliments témoins sans antibiotique de même composition conservés dans les mêmes conditions.

(1) Actuellement, cette préparation a été remplacée dans le commerce par un mélange de sels de calcium et de magnésium. 
Ces analyses étaient pratiquées après les intervalles de temps suivants (stades), désignés par les chiffres $I \grave{a} V: 5$ jours (délai nécessaire à la mise en route du premier dosage), I mois, 2 mois, 3 mois et 6 mois. Toutefois, par suite de difficultés matérielles, le dosage de la spiramycine n'a pas été effectué au stade III.

\section{TABIEAU I}

Formule et composition des aliments

\begin{tabular}{|c|c|c|c|c|}
\hline Aliment & $A$ & $\mathrm{~B}$ & $\mathrm{C}$ & D \\
\hline Céréales..$\ldots \ldots \ldots \ldots \ldots \ldots$ & 53 & 52 & 53 & 52 \\
\hline T. soja cuit $\ldots \ldots \ldots \ldots \ldots$ & 18 & 18 & 8 & 8 \\
\hline Farine de poisson Norvège.... & 12 & 12 & 6 & $\dot{b}$ \\
\hline Huile arachide $\left({ }^{1}\right) \ldots \ldots \ldots \ldots$ & 10 & 一 & 10 & - \\
\hline 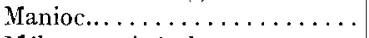 & 2 & 13 & 18 & 29 \\
\hline Mélange minéral $\ldots \ldots \ldots \ldots$ & 3 & 3 & $: 3$ & 3 \\
\hline \multirow[t]{2}{*}{ Prémélange antibiotique .... } & 2 & 2 & 2 & 2 \\
\hline & 100 & 100 & 100 & 100 \\
\hline Matière azotée brute p. 100. & 25 & 25 & 16 & 16 \\
\hline Lipides totaux p. $100 \ldots \ldots \ldots$ & 12 & 2 & 12 & 2 \\
\hline
\end{tabular}

(1) Stabilisée par 0,01 p. 100 de B. H. T.

TABLEAU 2

Conditions atmosphériques pendant la durée de l'expérience

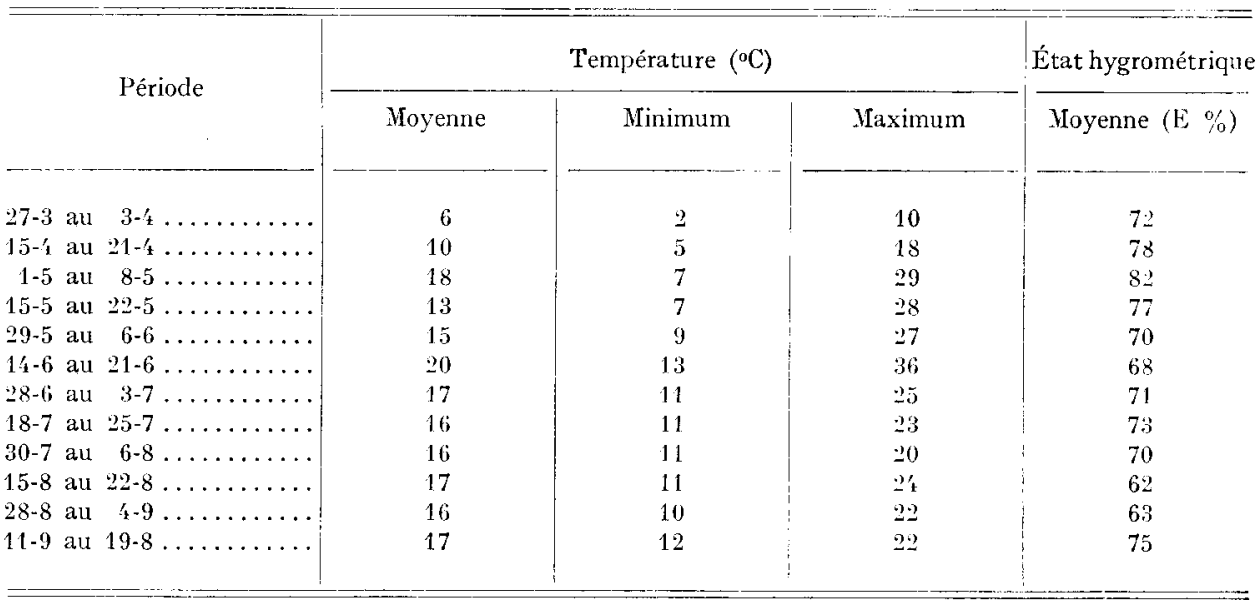

A chaque stade, deux échantillons moyens de $500 \mathrm{~g}$ de chaque aliment étaient prélevés et soumis simultanément pour analyse à deux laboratoires différents. Cette procédure d'analyse contradictoire était apparue nécessaire pour tenir compte de la variabilité des dosages et du nombre élevé de répétitions indispensable à la précision de chaque résultat.

Les techniques d'analyses étaient celles utilisées couramment par les différents laboratoires et décrites par Grove et Randall (1955), Analytical Comittee (1963) et Laboratoires Rhône-Poulenc 
(1966) : le principe est celui du disque de diffusion en culture sur plaque de gélose. Les germes utilisés étaient : B. Cereus mycoide. ATCC $11 \% 8$ (Pasteur) pour l'auréomycine et la terramycine et Sarcina lutea $A T C$ C 1341 (Pasteur) pour la spiramycine. Bien que les techniques utilisées par les laboratoirés pratiquant le même dosage aient été, en principe, les mêmes, de légères différences opératoires sont apparues (technique d'extraction, mode de culture, nombre de répétitions). Elles ne semblaient pas de nature ì modifier la validité des résultats et il n'en a pas été tenu compte dans les comparaisons. Par contre, une modification plus importante (augmentation du nombre d'extractions), proposée en cours d'expérience par l'un des laboratoires pour améliorer la récupération de certain. antibiotiques dans les aliments enrichis en lipides, n'a pu être prise en considération pour ne pas détruire l'homogénéité des résultats, ce (jui aurait rendu impossible toute interprétation pratique.

\section{d) Calculs}

Pour faciliter les calculs et permettre les comparaisons statistiques, tous les résultats ont été exprimés en p. 100 de la dose initiale, supposée exactement connue. I.e temps zéro de l'expérience était celui de l'introduction des antibiotiques dans la mélangeuse et bien que cette opération ait été exécutée avec beaucoup de précautions, il n'a pas été possible de vérifier si les concentrations initiales étaient bien celles qui étaient prévues.

Tous les résultats concernant un mème échantillon ont été regroupés. Une analyse de variance préliminaire a montré en effet qu'il n'y avait pas de différence significative entre les séries de résultats homologues des différents laboratoires. Les effets de la durée de conservation (5 stades), de la nature de l'antibiotique ( 3 produits), de la composition de l'aliment (4 formules), ainsi (que de la combinaison de la dose et du mode de présentation (4 cas) ont été analysés simultanément par la méthode des moindres carrés sur ordinateur IBM 1620 (programme 65025 de la S. C. G. A.). L'analyse a été poursuivie en considérant les facteurs 3 à 3 , puis 2 à 2 , afin de déterminer l'origine et l'importance des interactions de différents ordres. Les meilleures estimations des moyennes de dosages correspondant aux différents niveaux des 4 traitements ont été calculées dans l'hypothèse de non interaction des facteurs. Puis, après avoir été ordonnées, elles ont été comparées par la méthode de JUNCAN (1955).

\section{RÉSUL'TATS}

\section{Effets principaux}

Les tableaux 3,4 et 5 rapportent pour chacun des antibiotiques les valeurs moyennes des activités des différents échantillons correspondant aux 4 alinents. $336 \mathrm{r}$ résultats individuels ont été utilisés dans ces calculs (soit une moyenne de I4 répétitions par valeur exprimée).

L'analyse statistique des résultats individuels a montré que l'influence de tous les facteurs étudiés (stade de conservation, composition de l'aliment, antibiotique, dose et présentation) sur l'activité antibiotique des échantillons était hautement significative. Il est difficile d'apprécier l'importance de ces influences car, à l'exception de la dernière, les variances qui s'y rapportent ne diffèrent pas significativement entre elles. Sous réserve de 1'hypothèse de non-interaction, on peut cependant, sans grand risque d'erreur, les classer dans l'ordre des valeurs de F correspondant à chaque facteur (tabl. ()). I)ans ces conditions, la nature de l'antibiotique et la durée de conservation seraient les deux composantes principales de la stabilité ; la composition de l'aliment et sa présentation viendraient ensuite. $\mathrm{I}_{4}$ a concentration en antibiotiques aurait un moindre effet.

Avec les mêmes réserves, l'examen des moyennes par traitement (estimation par analyse simultanée des quatre facteurs) donne des renseignements complémentaires (tab1. 6). 
TABLEAU 3. - Activité antibiotique dans les aliments contenant de la chlortétracycline (p. 100 de la dose initiale)

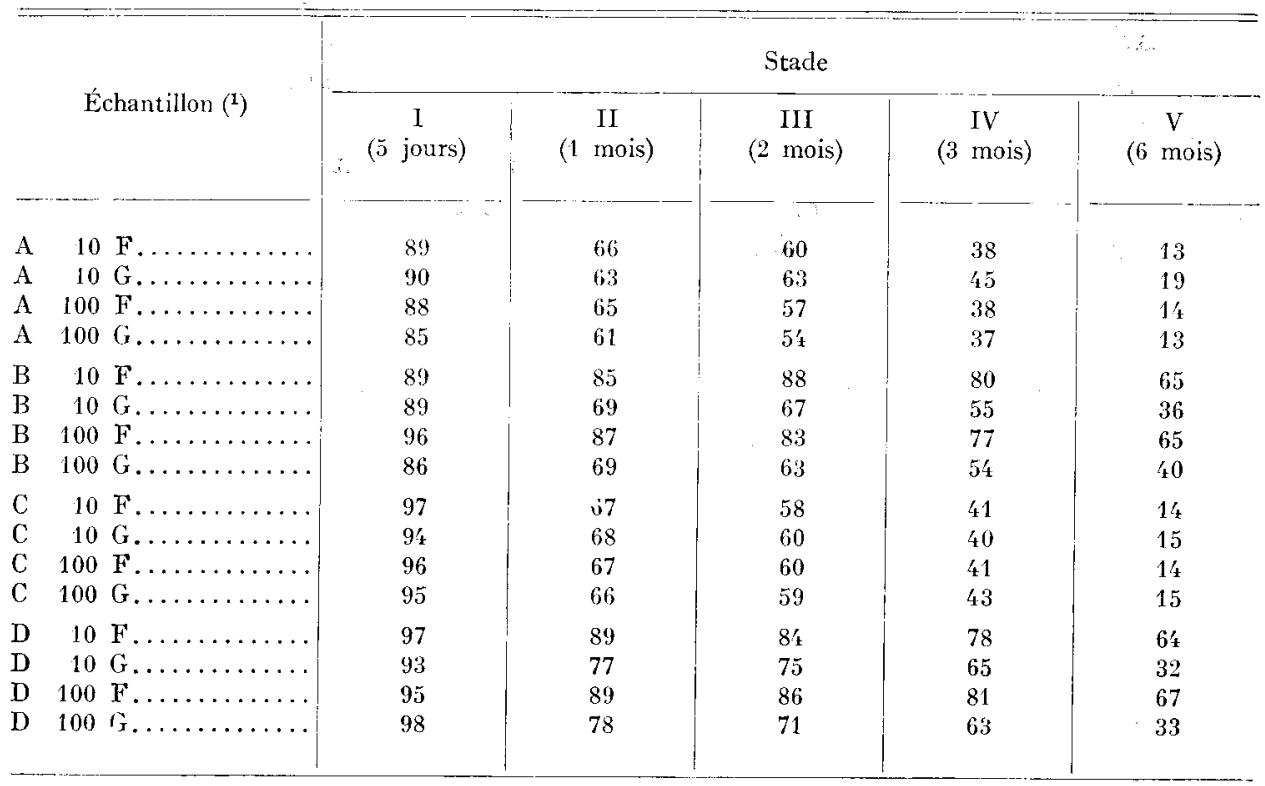

(1) A-B-C : aliments ; 10-100 : doses en Ppm; F-G : farine, granulé.

TABLEAU 4. - Activité antibiotique dans les aliments contenant de la spiramycine (p. roo de la dose initiale)

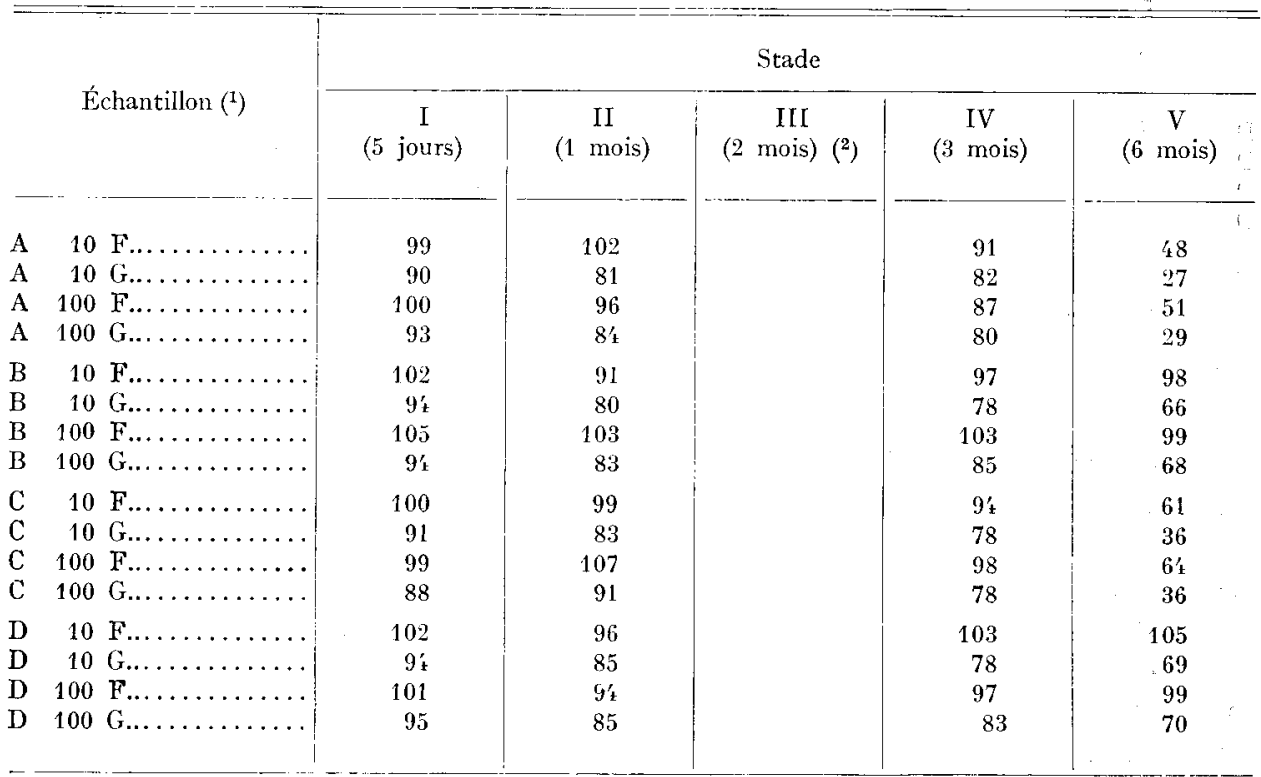

(1) A-B-C-D : aliments; 10-100: doses; F-G : farine, granulé.

$\left({ }^{2}\right)$ Dosages non effectués. 
A l'exception des aliments $B$ et $D$, toutes les différences entre traitements sont significatives. On peut alors faire les commentaires suivants :

- Antibiotique : la chlortétracycline a une stabilité moyenne inférieure d'environ 30 p. Ioo à celle des deux autres antibiotiques. Ces deux derniers diffèrent peu.

- Stade de conservation : les antibiotiques se dégradent rapidement dans les aliments au cours du stockage. Ce phénomène apparaît dès le premier mois, mais il s'accentue au cours des 3 derniers.

TABLEAU 5

Activité antibiotique dans les aliments contenant de l'oxytétracycline

(p. 100 de la dose initiale)

\begin{tabular}{|c|c|c|c|c|c|c|}
\hline \multirow{2}{*}{\multicolumn{2}{|c|}{ Échantillon ( $\left.{ }^{(}\right)$}} & \multicolumn{5}{|c|}{ Stade } \\
\hline & & $\left(\begin{array}{c}\mathrm{I} \\
\text { (5ours) }\end{array}\right.$ & (1 $\begin{array}{l}\text { II } \\
\text { mois })\end{array}$ & $\left(\begin{array}{l}\text { III } \\
\text { mois) }\end{array}\right.$ & $\left(\begin{array}{l}\text { IV } \\
\text { mois })\end{array}\right.$ & $\begin{array}{c}\mathrm{V} \\
(6 \mathrm{mois})\end{array}$ \\
\hline A & $10 \mathrm{~F} \ldots \ldots \ldots \ldots$ & 95 & 91 & 87 & 85 & 62 \\
\hline A & $10 \mathrm{G} \ldots \ldots \ldots \ldots \ldots$ & 99 & 96 & 85 & 91 & 71 \\
\hline A & $100 \mathrm{~F} \ldots \ldots \ldots \ldots \ldots$ & 93 & 85 & $8: 2$ & 77 & 62 \\
\hline$A$ & $100 \mathrm{G} \ldots \ldots \ldots \ldots$ & 97 & 83 & 73 & 69 & 58 \\
\hline B & $10 \mathrm{~F} \ldots \ldots \ldots \ldots$ & $10^{\prime}$ & 101 & 98 & 99 & 89 \\
\hline B & $10 G \ldots \ldots \ldots \ldots$ & 100 & 96 & 91 & 88 & 79 \\
\hline $\mathrm{B}$ & $100 \mathrm{~F} \ldots \ldots \ldots \ldots$ & 101 & 99 & $9:$ & 89 & 80 \\
\hline B & $100 \mathrm{G} \ldots \ldots \ldots \ldots \ldots$ & 93 & 86 & 86 & $8: 2$ & 70 \\
\hline $\mathrm{C}$ & $10 \mathrm{~F} \ldots \ldots \ldots \ldots$ & 96 & 92 & 87 & 78 & 62 \\
\hline $\mathrm{C}$ & $10 \mathrm{G} \ldots \ldots \ldots \ldots$ & 103 & 96 & 91 & 86 & 70 \\
\hline $\mathrm{C}$ & $100 \mathrm{~F} \ldots \ldots \ldots \ldots$ & 95 & 98 & $8^{\prime} t$ & 79 & 59 \\
\hline $\mathrm{C}$ & $100 \mathrm{G} \ldots \ldots \ldots$ & 95 & 88 & 80 & 74 & 61 \\
\hline $\mathrm{D}$ & $10 \mathrm{~F} \ldots \ldots \ldots \ldots$ & 99 & 97 & 103 & 99 & 87 \\
\hline $\mathrm{D}$ & $10 \mathrm{G} \ldots \ldots \ldots$ & 97 & 90 & 91 & 87 & 77 \\
\hline $\mathrm{D}$ & $100 \mathrm{~F} \ldots \ldots \ldots$ & 98 & 91 & 91 & 98 & 90 \\
\hline D & $100 \mathrm{G} \ldots \ldots \ldots \ldots \ldots$ & 93 & 93 & 91 & 81 & 76 \\
\hline
\end{tabular}

(1) A-B-C-D : aliments ; 10-100: doses ; F-G : farine, granulé.

- Composition de l'aliment : l'addition d'un taux élevé de graisse aux aliments (A et C) diminue l'activité antibiotique d'environ $20 \mathrm{p}$. Ioo par rapport aux aliments sans graisse (B et D). Par contre, l'augmentation du taux de protéine (aliments $A$ et $B$ ) ne modifie pas cette activité par rapport aux aliments à taux de protéine plus faible (C et D).

- Mode de présentation de l'aliment: l'influence de la granulation est nette (aliments Io $\mathrm{G}$ et roo $\mathrm{G}$ ), elle entraîne une perte moyenne de $\mathrm{x} 2$ p. Ioo du pouvoir antibiotique, par rapport aux aliments présentés en farine (Io $\mathrm{F}$, Ioo F).

- Dose d'antibiotique: la conservation de l'antibiotique semble légèrement moins bonne ( 2 p. IOO) aux concentrations fortes (IOO (', IOO F) qu'aux faibles concentrations (Io $\mathrm{F}$, Io $\mathrm{G}$ ). 


\section{TABLEAU 6}

Influence des différents traitements sur la stabilité des antibiotiques

\begin{tabular}{|c|c|c|c|c|}
\hline Facteur étudié & Antibiotique & Stade & $\begin{array}{l}\text { Composition } \\
\text { de l'aliment }\end{array}$ & $\begin{array}{c}\text { Dose } \\
\text { Mode de } \\
\text { présentation }\end{array}$ \\
\hline Valeur moyenne du traitement & $\begin{array}{l}\mathrm{Cl}=63,38 \\
\mathrm{Ox}=87,10 \\
\mathrm{Sp}=86,03\end{array}$ & $\begin{aligned} \mathrm{I} & =96,73 \\
\mathrm{II} & =85,28 \\
\mathrm{III} & =81,07 \\
\mathrm{IV} & =75,11 \\
\mathrm{~V} & =56,50\end{aligned}$ & $\begin{array}{l}\mathrm{A}=70,08 \\
\mathrm{~B}=86,06 \\
\mathrm{C}=72,88 \\
\mathrm{D}=86,75\end{array}$ & $\begin{array}{r}10 \mathrm{~F}=84,18 \\
100 \mathrm{~F}=83,09 \\
10 \mathrm{G}=75,86 \\
100 \mathrm{G}=72,63\end{array}$ \\
\hline $\begin{array}{l}\text { Valeur de } \mathrm{F} \\
\text { pour le facteur considéré } \\
\text { (degrés de liberté } n_{1} / n_{2} \text { ) }\end{array}$ & $\begin{array}{c}1195^{* *} \\
(2 / 3137)\end{array}$ & $\begin{array}{c}995 * * \\
(4 / 3117)\end{array}$ & $\begin{array}{c}362^{* *} \\
(3 / 3117)\end{array}$ & $\begin{array}{c}142 * * \\
(3 / 3117)\end{array}$ \\
\hline $\begin{array}{l}\text { Valeur de la plus petite } \\
\text { différence significative } \\
(\mathrm{P}<0,01)\end{array}$ & 0,92 & 1,18 & 1,05 & 1,05 \\
\hline
\end{tabular}

\section{Abréviations :}

$\mathrm{Cl}$ : chlortétracycline; $\mathrm{Ox}$ : oxytétracycline; $\mathrm{Sp}:$ spiramycine,

I : 5 jours; II : 1 mois ; III : 2 mois; IV : 3 mois ; $\mathrm{V}: 6$ mois.

A : 10 p. 100 lipides +25 p. 100 protides; B : 25 p. 100 protides ; C : 10 p. 100 lipides +14 p. 100 protides; $\mathrm{D}: 15 \mathrm{p} .100$ protides.

$10 \mathrm{~F}: 10$ p.p.m./farine ; $100 \mathrm{~F}: 100$ p.p.m./farine

$10 \mathrm{G}: 10$ p.p.m. /granulés ; $100 \mathrm{G}: 100$ p.p.m./granulés.

\section{TABLEAU 7}

Valeurs de $F$ (et degrés de liberté) pour les interactions entre les différents facteurs agissant sur la stabilité des antibiotiques

\begin{tabular}{|c|c|c|c|c|c|c|}
\hline \multirow[b]{2}{*}{ Interaction } & \multicolumn{2}{|c|}{ 1er ordre } & \multicolumn{2}{|c|}{$2^{\mathrm{e}}$ ordre } & \multicolumn{2}{|c|}{$3^{\mathrm{e}}$ ordre } \\
\hline & $\mathrm{F}$ & $\begin{array}{l}\text { degrés de } \\
\text { liberté }\end{array}$ & $\mathrm{F}$ & $\begin{array}{l}\text { degrés de } \\
\text { liberté }\end{array}$ & $\mathrm{F}$ & $\begin{array}{c}\text { degrés de } \\
\text { liberté }\end{array}$ \\
\hline Stade / antibiotique & 73,7 & 7 & & & & \\
\hline Stade / aliment $\ldots \ldots \ldots \ldots \ldots \ldots$ & 29,3 & 12 & & & & \\
\hline Antibiotique / forme ........... & 11,4 & 6 & & & & \\
\hline Antibiotique / aliment. ..... & 4,9 & 12 & & & & \\
\hline Stade / forme ....... & 3,4 & 12 & & & & \\
\hline Stade / antibiotique / aliment .... . & & & 48,8 & 46 & & \\
\hline Stade / antibiotique / forme... & & & 19,5 & 46 & & \\
\hline Stade / aliment / forme........... & & & 8,1 & 69 & & \\
\hline Aliment / antibiotique / forme.... & & & 5,5 & 39 & & \\
\hline Stade / antibiotique / aliment / forn & & & & & 24,8 & 211 \\
\hline
\end{tabular}

(1) Degrés de liberté de la variance résiduelle 3305 à 33 1. 


\section{Interactions}

Le tableau 7 rapporte les valeurs du test $F$ concernant les interactions possibles entre les divers facteurs considérés 2 à 2,3 à 3 , ou tous simultanément. Toutes ces interactions sont significatives avec de légères différences qui permettent (pour un même nombre de degrés de liberté) d'en apprécier l'importance relative. Il en résulte que la stabilité d'un antibiotique dans un aliment dépend simultanément, mais à des degrés variables, de tous les facteurs considérés. Il n'est donc pas possible d'en tirer de conclusions générales et il faut, au contraire, considérer chaque cas particulier. Nous nous sommes limités à examiner les interactions les plus intéressantes.

\section{a) Stade / antibiotique / aliment.}

La figure I montre que la stabilité des antibiotiques au cours de la conservation varie avec la nature de l'antibiotique (interaction du $I^{\text {er }}$ ordre) : 1'oxytétracycline présente une perte d'activité régulière, sensiblement proportionnelle au temps de stockage; la chlortétracycline se dégrade proportionnellement beaucoup plus vite (30 p. IOo) au cours du premier mois de conservation; la spiramycine se conserve relativement bien pendant les 3 premiers mois, mais parait plus fragile ensuite.

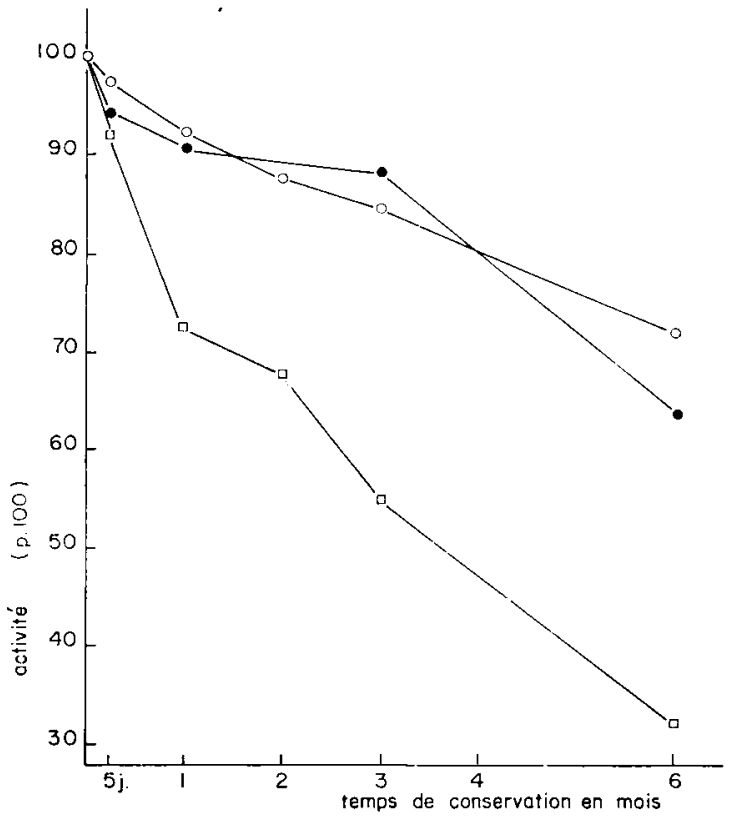

Fig. I. - Évolution de l'activité movenne des différents antibioliques dans les aliments

C Chlortétracycline

O Oxytétracycline

- Spiramycine

Mais ce schéma peut être nuancé en fonction des aliments utilisés (interaction du $2^{\mathrm{e}}$ ordre). Les figures $2 a, 2 b$ et $2 b$ montrent que suivant qu'il s'agit d'aliment enrichi ou non en graisse, la perte d'activité des différents antibiotiques n'est pas la même : pour la chlortétracycline, l'influence de la graisse est importante et apparaît 
dès le premier mois (fig. $2 a$ ) ; pour l'oxytétracycline, l'effet des lipides n'apparaît qu'au bout de deux mois. Pour la spiramycine, la perte de stabilité des antibiotiques dans les aliments gras n'est importante qu'après 3 mois.

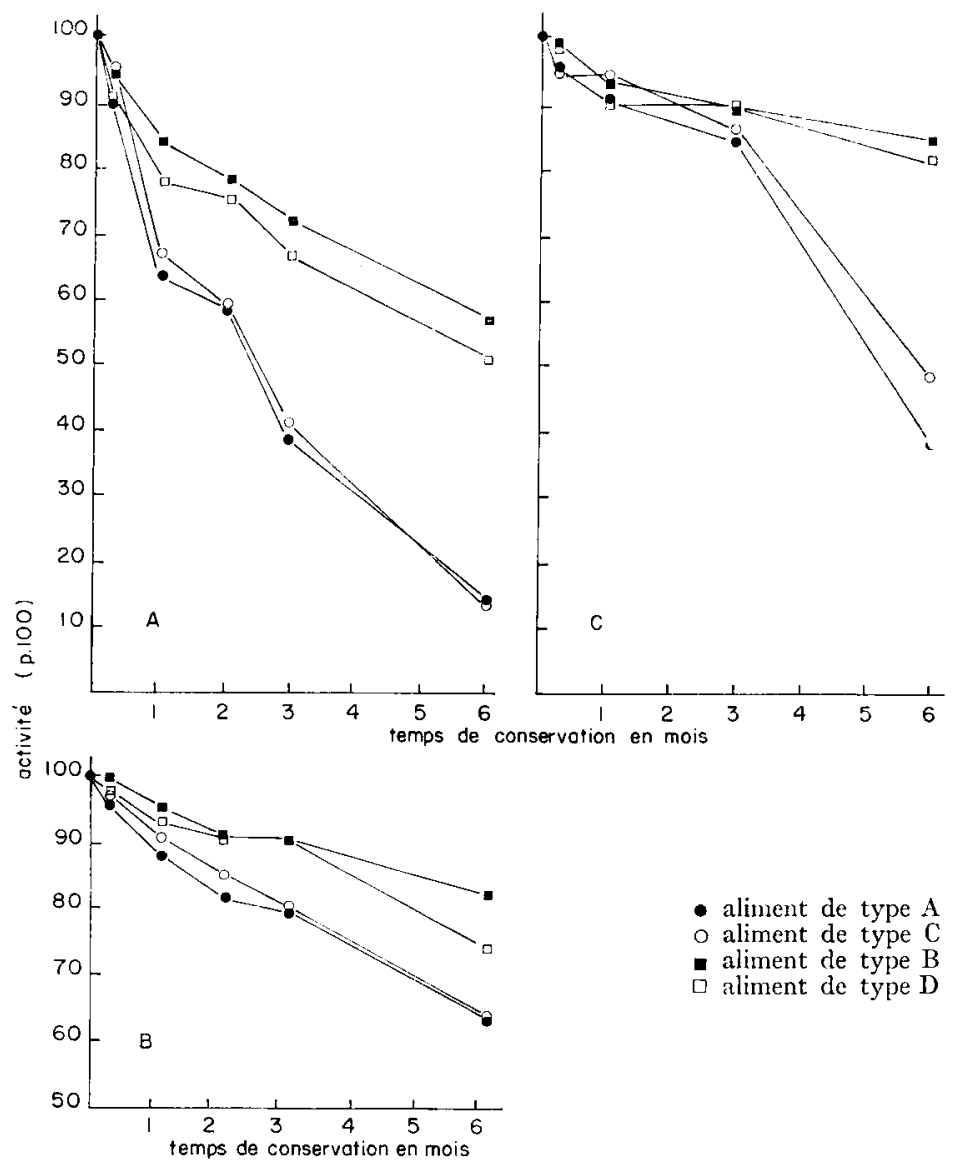

FIG. 2. - Évolulion de l'activilé des antibiotiques dans lifférenls aliments $a$ - Chlortétracycline $\quad b-$ Oxytétracycline $\quad c-$ S s iramycine

b) Stade / antibiotique / forme.

I a granulation entraîne une perte d'activité de l'antibiotique, par rapport à la présentation en farine. Ce phénomène s'intensifie avec le temps (interaction du $I^{e r}$ ordre), mais il se manifeste différemment suivant les antibiotiques (interaction du $2^{\mathrm{e}}$ ordre). La figure 3 rapporte l'évolution de l'activité antibiotique des aliments en fonction du mode de présentation et de l'antibiotique utilisé. L'effet maximum apparaît dans les aliments supplémentés à la spiramycine : la perte d'activité due à la granulation est importante dès le pressage et atteint $40 \mathrm{p}$. roo au bout de 6 mois. Avec la chlortétracycline, la perte est relativement moins importante et augmente progressivement jusqu'à I5 p. Ioo en fin de stockage. L'oxytétracycline supporte encore mieux la granulation des aliments : la perte d'activité n'apparaît qu'après 2 mois et ne dépasse pas 7 p. roo en moyenne. 


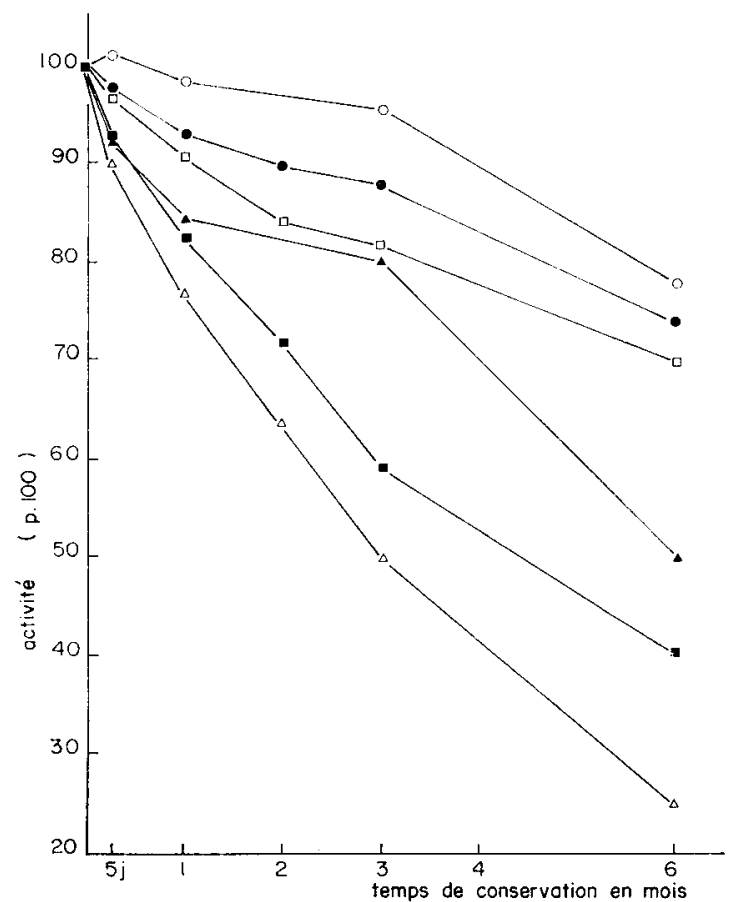

FIG. 3. - Évolution de l'activité des différents antibiotiques suivant le mode de présentation des aliments

o spiramycine, farine A spiramycine, granulé
- oxytétracycline, farine

¿ oxytétracycline, granulé
- chlortétracycline, farine $\Delta$ chlortétracycline, granulé

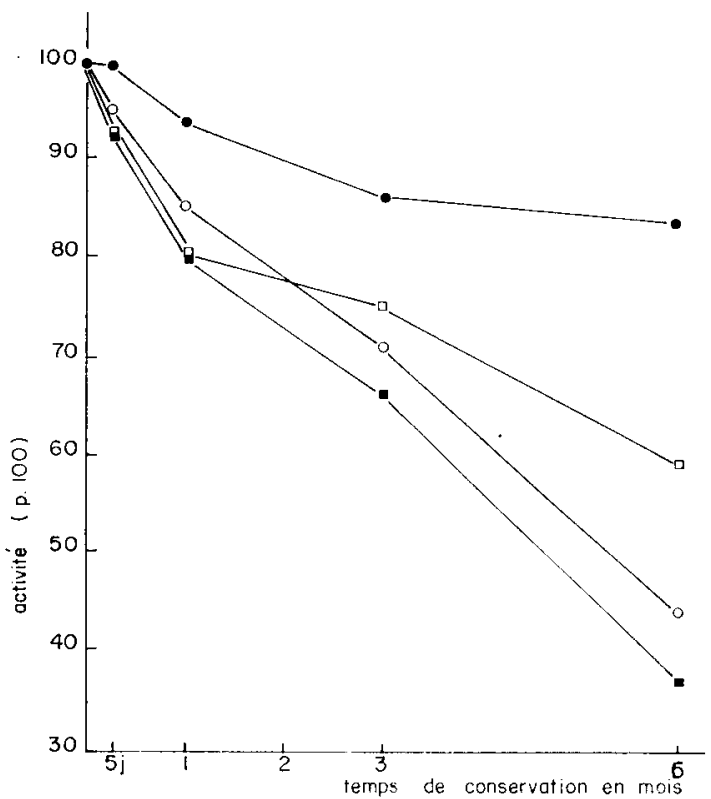

FIG. 4. - Évolution de l'activité antibiotique suivant le mode de présentation et la composition des aliments

- aliments sans graisse $(B, D)$, farine

$\square$ aliments sans graisse (B, D), granulé $\circ$ aliments avec graisse $(A, C)$, farine - aliments avec graisse $(A, C)$, granulé 
c) Interaction aliment / forme / stade / antibiotique.

I a granulation, comme la présence de graisse dans l'aliment, diminue la stabilité des antibiotiques au cours de la conservation. On pourrait penser que les effets de ces deux facteurs se cumulent dans les combinaisons où elles se rencontrent (interaction du $2^{\mathrm{e}}$ ordre). Il semble qu'il n'en soit rien.

La figure 4 montre que l'effet de la granulation est plus important dans les aliments sans graisse $(\mathrm{B}, \mathrm{D})$, dans lesquels la perte d'activité atteint $30 \mathrm{p}$. Ioo à 6 mois, que dans les aliments gras (A. C) où cette perte d'activité reste sensiblement constante et ne dépasse pas ro p. Ioo.

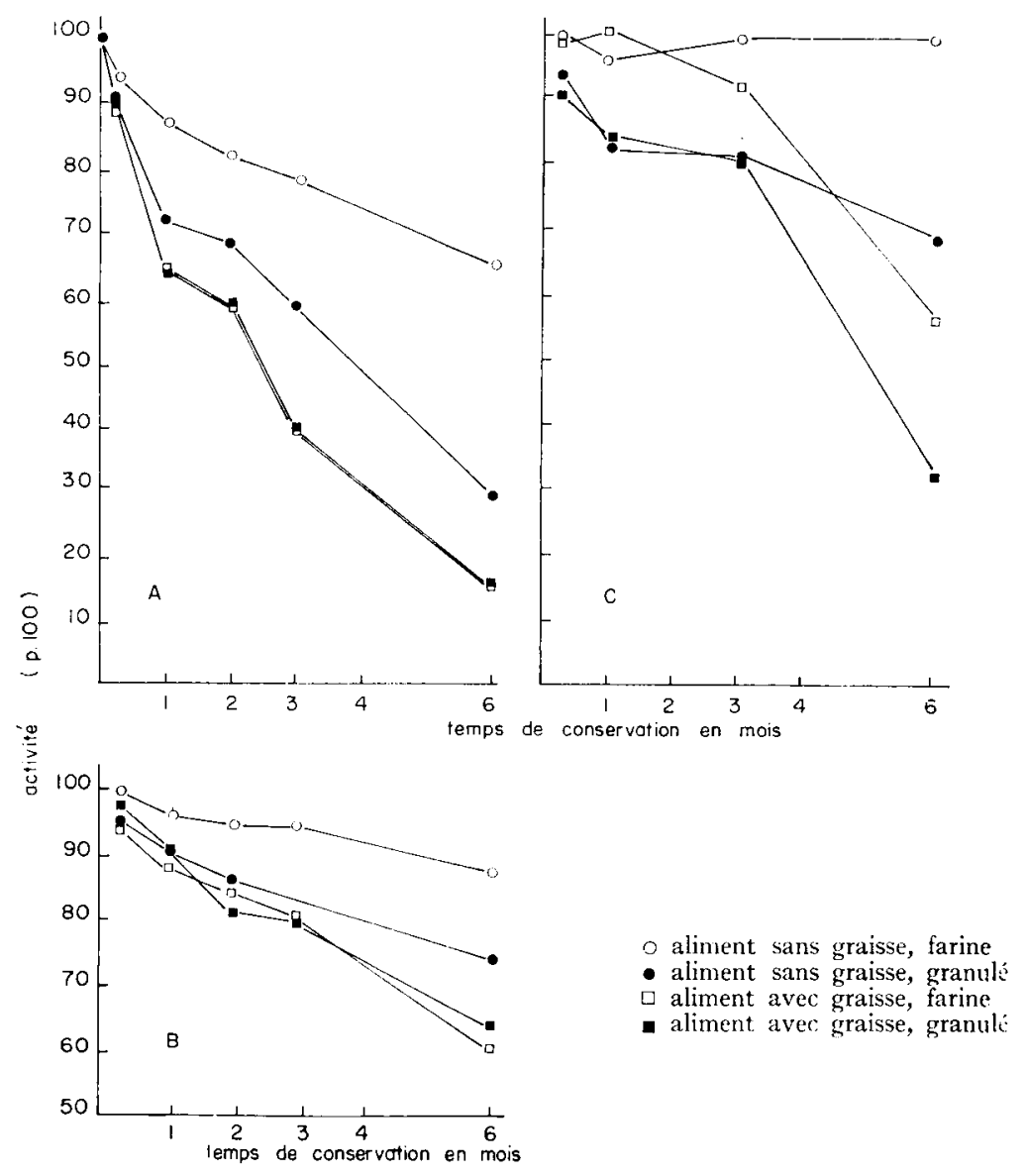

FIG. 5. - Évolution de l'activité des différents antibiotiques suivant le mode de présentation et la composition des aliments

$a$ - Chlortétracycline $\quad b$-Oxytétracycline $\quad c$ - Spiramycine

Mais ce phénomène est aussi variable selon l'antibiotique (interaction du $3^{\mathrm{c}}$ ordre) : dans les aliments supplémentés à l'oxytétracycline et à la chlortétracycline, l'effet de la granulation est rigoureusement nul lorsque ceux-ci sont enrichis en graisse, 
alors qu'il est très important dans les aliments sans graisse (fig. $5 a$ et $5 b$ ). Par contre, avec la spiramycine, la présence de graisse n'empêche pas la dégradation de l'antibiotique due à la granulation (fig. $5 \mathrm{c}$ ).

d) Dose / antibiotique / aliment.

Seule l'oxytétracycline semble se dégrader nettement plus vite ( 8 p. Ioo) à dose élevée qu'à dose ráible dans les aliments. Le phénomène est surtout apparent avec les aliments $A$ et $B$, c'est-à-dire avec ceux qui contiennent le plus de protides (tab1. 8). La chlortétracycline réagit dans le même sens mais d'une façon moins marquée et la spiramycine dans le sens contraire.

TABLEAU 8

Influence de la dose d'antibiotique sur la stabilité des différents antibiotiques dans les aliments

\begin{tabular}{|c|c|c|c|c|c|c|}
\hline \multirow{2}{*}{$\frac{\text { Antibiotique } \ldots \ldots \ldots \ldots \ldots}{\text { Dose antibiotique (p.p.m.) } \ldots \ldots}$} & \multicolumn{2}{|c|}{ Oxytétracycline } & \multicolumn{2}{|c|}{ Chlortétracycline } & \multicolumn{2}{|c|}{ Spiramycine } \\
\hline & 10 & 100 & 10 & 100 & 10 & 100 \\
\hline$A, \ldots \ldots \ldots \ldots \ldots$ & 86 & 78 & 55 & 51 & 76 & 78 \\
\hline \multicolumn{7}{|l|}{ Aliments $\quad B \ldots \ldots \ldots \ldots \ldots \ldots$} \\
\hline $\mathrm{C} \ldots \ldots \ldots \ldots \ldots$ & 86 & 82 & 55 & 55 & 80 & 81 \\
\hline$D \ldots \ldots \ldots \ldots \ldots$ & 93 & 90 & 75 & 75 & 91 & 94 \\
\hline Moyenne ............. & 90 & $8:$ & 64 & 63 & 84 & 86 \\
\hline
\end{tabular}

\section{DISCUSSION}

Il est permis de faire des réserves sur la valeur des dosages microbiologiques pour mettre en évidence les altérations supportées par les antibiotiques dans les aliments et l'incidence de ce phénomène sur la réponse des animaux. Toutefois, le fait qu'on ait trouvé une bonne concordance entre ces dosages et l'analyse chimique, qui, dans des conditions analogues, fait apparaître une dégradation importante des antibiotiques par épimérisation, lève en grande partie ce doute (KATZ, I963; KATz et FAssBENDER, I 967 ). Nos résultats confirment, en outre, un certain nombre de faits qui apparaissaient déjà avec divers antibiotiques, aussi bien en produits purs, qu'en solution ou dans des préparations pharmaceutiques ou alimentaires : influence du substrat de la présentation, du temps de conservation (WoRNICK, Ig67).

Malgré les interférences rencontrées, nous pouvons donc essayer de dégager le rôle des principaux facteurs examinés :

\section{a) Nature de l'antibiotique}

Les valeurs moyennes dont nous disposons se rapportent à 3 antibiotiques différents, dont la forme chimique, elle-même, est différente. Flles n'ont qu'une signification limitée, car elles ne correspondent à aucune condition précise (stade moyen). 
Elles suffisent cependant à montrer que des différences de stabilité importantes peuvent apparaître entre les antibiotiques lorsqu'ils sont incorporés aux aliments. Ces différences sont de l'ordre de celles que rapporte WOR.NICK (I959) avec divers antibiotiques, dont l'érythromycine. llans des conditions similaires, KOROLEVA et Penova (I963) avaient conclu que les tétracyclines se conservaient beaucoup mieux que diverses préparations de pénicilline. Dans le cas présent, la stabilité de la chlortétracycline est beaucoup moins bonne que celle de la spiramycine, elle-même légèrement inférieure à celle de 1'oxytétracycline, résultat qui confirme les observations de WEICH (I950), I)IDING (I954) et KaWABATA et al. (I960).

Il ne peut être question de rattacher trop étroitement les différences de stabilité que nous avons observées aux formes chimiques utilisées. On sait cependant que celles-ci ont une grande importance (MUI, LER et al., I958; WoRNICK et KUHN, I962). Le fait que certaines expériences aient montré que l'oxytétracycline et la chlortétracycline conservaient, sous une même forme chlorhydrate, une activité comparable (Kororeva et PEHOVA, I965) conduirait à penser que la différence de forme sous laquelle ces mêmes antibiotiques ont été comparés dans notre expérience n'est peutêtre pas étrangère aux résultats obtenus.

\section{b) Temps de conservation}

I a dégradation du pouvoir antibiotique au cours de la conservation est classique, nuais elle peut être plus ou moins rapide suivant les conditions (WorNICK, I967 ; KAWABATA et al., I960). La dégradation de la chlortétracycline est très brutale,

TABLEAU 9

Durée limite de conservation (mois) des aliments de différents types pour une activité antibiotique minimum de 75 et $50 \mathrm{p} .100$ de la dose initiale

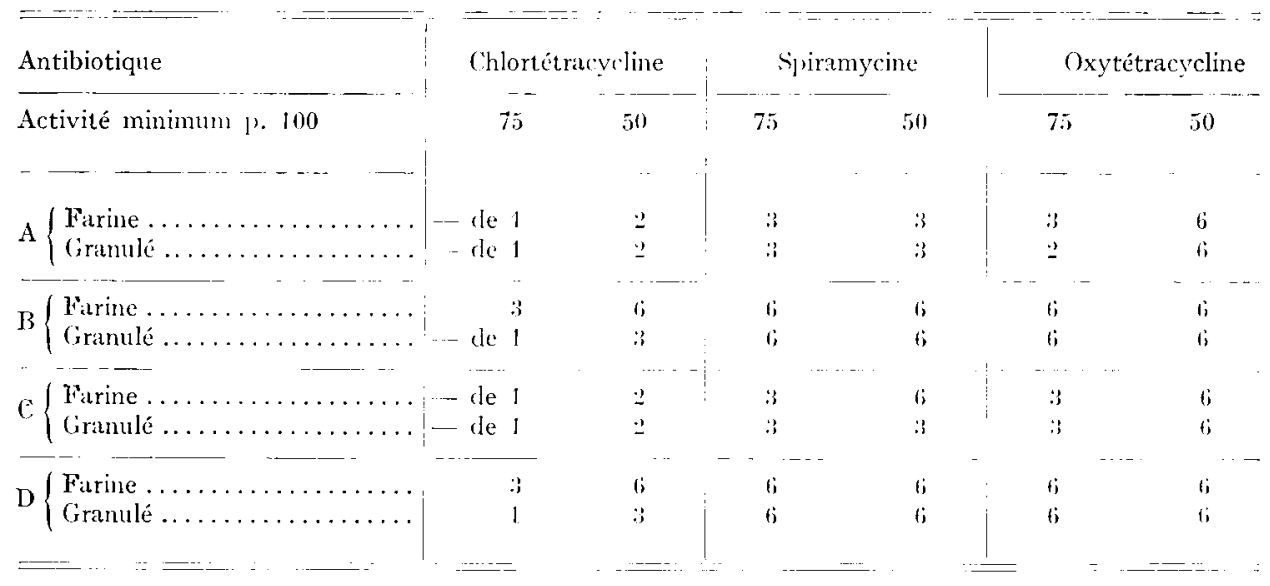

quels que soient les aliments utilisés. Celles de la spiramycine et de l'oxytétracycline sont, en comparaison, nettement moins rapides, surtout dans les aliments ne contenant pas de graisse et présentés en farine. Pour ces deux antibiotiques, la perte d'activité après 6 mois de conservation atteint cependant en moyenne 30 à $40 \mathrm{p}$. Ioo de la dose initiale, ce qui est du même ordre que la valeur trouvée pour la bacitracine 
dans les aliments pour volailles (HusAAs, I967). Mais le phénomène varie aussi beaucoup suivant les échantillons. Il en résulte que si les fabricants d'aliments devaient donner une garantie de conservation pour les antibiotiques qu'ils utilisent, celle-ci devrait varier non seulement avec l'antibiotique et le taux de conservation considéré comme minimum acceptable (75 ou 50 p. Ioo), mais aussi avec le type d'aliment (tab1. 9).

\section{c) Conditions atmosphériques}

Il ne semble pas que, dans le cadre de cette expérience (aliments conservés en sacs sous abri), les conditions atmosphériques soient intervenues d'une façon appréciable sur la stabilité des antibiotiques. On sait cependant que de faibles variations de température peuvent accélérer les phénomènes d'épimérisation des antibiotiques (KATZ et FASSBENDER, I967). Paradoxalement, on constate que la baisse d'activité relativement importante supportée par tous les aliments pendant le premier mois est apparue au moment où la température extérieure était la plus basse. Inversement, la température et 1'humidité plus élevées au cours des mois suivants ne paraissent pas avoir accéléré la dégradation des antibiotiques, sauf peut-être dans les aliments qui contenaient de la graisse (fig. $2 a, 2 c$ ).

\section{d) Composition des aliments}

Les constituants chimiques des aliments capables de modifier l'activité des antibiotiques sont nombreux. Les plus connus sont les sels minéraux, qui ont suscité un grand nombre de travaux (ArBERT, I953; PRICE et al., I957; BINKLEY, I955; HARMs et al., I964; WEINBERG, I957), et les vitamines (GEIGER, I946; WORNICK, I967).

I'influence des substances azotées est plus discutée. Pour certains auteurs, les protides accélèrent la dégradation (WEINSTFIN, I958 ; PRICE et coll., I957), pour d'autres, ils la diminuent (KAWABATA et al., I960). Notre expérience ne nous permet pas de trancher, car si pour l'oxytétracycline l'élévation du taux de protides de l'aliment a légèrement accru les pertes en antibiotique, cet effet est apparu tardivement et n'est pas très important. Il est en outre négligeable pour les deux autres antibiotiques, résultat conforme à celui trouvé par KATZ et FASSBENDER (I967) avec la chlortétracycline.

L’influence de la graisse du régime a été peu étudiée jusqu'à présent (WHITTET, I959). Certains auteurs ont cependant montré que la présence d'acides gras insaturés ou de peroxydes pouvait accélérer la dégradation des antibiotiques (WASKMAN, I950 ; Fox, r965), phénomène que confirme notre expérience. Cette influence est plus importante pour la chlortétracycline que pour la spiramycine ou l'oxytétracycline, ce qui peut être lié à la plus grande sensibilité de la première à l'épimérisation (KATz et FASSBENDER, I967). Un autre point aussi devra être éclairci, celui des difficultés d'extraction : après plusieurs mois de conservation, des difficultés de dosage des antibiotiques sont apparues pour certains antibiotiques dans les aliments enrichis en graisse, phénomène que WORNICK (I959) signale également. Une technique d'extraction plus poussée permettrait, dans certains cas, de récupérer une fraction supplémentaire de 1'activité initiale. Le problème est posé de savoir si cette activité qui échappe à l'analyse classique conserve une signification biologique. Dans le cas présent, ceci ne change rien aux conclusions précédentes. 


\section{e) Mode de présentation}

L'effet de la granulation sur l'efficacité alimentaire des constituants des aliments (amidon, protéines, vitamines) a été souvent étudié. Dans la plupart des cas, cette action peut s'expliquer par l'effet thermique auquel est soumis l'aliment au moment du pressage. Les antibiotiques n'échappent pas à ce dernier phénomène. La perte d'activité antibiotique que nous avons observée dans les aliments granulés est comparable à celle que signalent d'autres auteurs (WORNICK, I959-I962 ; STOCKSTAD, I952), elle varie de 7 à $4^{\circ}$ p. Ioo suivant les antibiotiques. Certains antibiotiques, comme l'oxytétracycline, y sont peu sensibles (peut-être en raison d'une meilleure stabilité à la chaleur), alors que d'autres, comme la spiramycine, sont beaucoup plus dégradés. Cet effet thermique semble confirmé par le fait que la présence de graisses dans les aliments limite beaucoup l'altération due au pressage. Par contre, la dégradation est maximum et s'aggrave avec le temps dans les aliments ne contenant pas de graisse (fig. 5 b). Il est, en effet, connu que la présence de corps gras facilite les opérations de pressage en diminuant l'échauffement et la dureté des granulés. Ceci ne compense qu'en partie cependant l'effet dépressif joué par certaines graisses sur la stabilité des antibiotiques et il reste à savoir si ce rôle protecteur lors du pressage ne pourrait être obtenu avec un moindre taux de graisse, ou éventuellement avec d'autres constituants (glycérol, vapeur d'eau).

\section{f) Dose d'antibiotique}

La tendance que l'on observe pour l'oxytétracycline à se dégrader davantage à concentration élevée (IOo p.p.m.) qu'à dose faible (Io p.p.m.) peut paraître peu importante. La cause ne nous en est d'ailleurs pas connue. Il faut toutefois remarquer que cette perte devient plus sensible sur le plan économique lorsqu'elle est exprimée en valeur absolue (p.p.m.). Dans les échantillons contenant ro p.p.m. au départ, la perte reste faible ( $\mathrm{x}$ p.p.m. en moyenne), mais elle atteint I6 p.p.m. dans les échantillons qui en contenaient roo. Cette perte peut alors dépasser le seuil de réponse des animaux et conduire à utiliser des doses inutilement élevées d'antibiotiques. I'antibiosupplémentation à dose élevée entraîne un gaspillage plus important et coûte donc proportionnellement plus cher que celle à dose faible. Sous cet aspect, ceci peut justifier l'emploi simultané de plusieurs antibiotiques différents à dose restreinte plutôt qu'un seul antibiotique à dose massive.

\section{CONCLUSION}

Les principaux résultats de cette expérience peuvent se résumer comme suit:

I. Les aliments antibiosupplémentés peuvent perdre une fraction importante de leur activité, variable jusqu'à 87 p. Ioo suivant les conditions expérimentales au cours d'une conservation de 6 mois.

2. La chlortétracycline est, en moyenne, moins stable dans les aliments que l'oxytétracycline ou la spiramycine (qualité « alimentation animale »). 
3. I a dégradation des antibiotiques est importante (surtout pour la chlortétracycline) dès le premier mois de conservation. I a demi-vie (efficacité 50 p. roo) est atteinte avant 2 mois pour la chlortétracycline, entre 3 et 6 mois pour la spiramycine et après 6 mois pour l'oxytétracycline.

4. I,es variations météorologiques saisonnières, agissent peu sur la conservation des antibiotiques dans les aliments stockés sous abri.

5. L'addition de graisse (huile d'arachide) aux aliments diminue la stabilité des antibiotiques, principalement celle de la chlortétracycline (après un mois) et de la spiramycine (après 3 mois).

6. Les variations du taux de protéines des aliments ne semblent pas modifier d'une façon importante la stabilité des antibiotiques.

7. La granulation des aliments provoque une perte moyenne d'activité antibiotique de 12 p. Ioo par rapport à la forme farine. Cet effet est maximum dans les aliments supplémentés à la spiramycine (30 p. I0o). Il apparaît surtout dans les aliments ne contenant pas de graisse.

8. En valeur relative, la perte d'activité des antibiotiques varie peu, sauf pour l'oxytétracycline, avec la dose d'antibiotique utilisée. İn valeur absolue, cette perte peut dépasser, quel que soit l'antibiotique, 'e seuil d'efficacité dans les aliments contenant des doses élevées d'antibiotiques.

9. Ces conclusions, valables pour des aliments destinés aux porcelets, ont été obtenues à l'aide de tests microbiologiques (in vitro). Il reste à les confirmer par des tests sur animaux d'élevage.

Rę̧u pour publication en mars 1968.

\section{REMMERCIEMENTS}

Cette étude a pu être menée à bien grâce à la collaboration des lítablissements Rhônc-P'oulenc (C. Roussel) et de la tociété industrielle de Biochimie (B. GODEFroy). Une partie importante des dosages nécessaires a été effectuées dans leurs laboratoires respectifs de Vitry (M. Dubost) et d'Amboise (J.-P. RAyNaud), ainsi qu'au Laboratoire d'analyse et d'essai des aliments de l'I. N. R. A. (J. J)elort-Laval et P. Valdebouze). L'exploitation statistique a été effectuée grâce à l'atelier mécanographique de la Station centrale de Génétique animale et nous avons bénéficié, pour l'interprétation des résultats, dés conseils de notre collègue C. ILGAULt. Nous leur adressons à tous nos plus rifs remerciements.

\section{SUMMARY}

STEIHES ON TIIE STABILITY OF ANTIBIOTICS IN PIGLET FEFISS

The stability of antibiotics through a six months storage was studied in 48 feed combinations. The factorial design covered : 3 antibiotics $\times 2$ doses (10-100 p.p.m. $\times 2$ protein level (16-25 p. 100) $\times 2$ lipids level (o-1o p. 100) $\because 2$ forms (meal, pellet).

Results, expressed is percent recovery estimated from microbiological activity varied from 13 to ro5 p. roo and are summarized in table 6. Conclusions may be as follows :

I. Chlortetracyclin is less stable $(63 \mathrm{p} .100$ in average) than oxytetracyclin ( $87 \mathrm{p}, 100)$ and spiramycin (86 p. ioo).

2. The maxinum rate of decomposition is achieved during the first month of storage (fig. $\mathrm{r}$ ). 
3. I.osses are higher in fat (ground nutoil) supplemented feeds, mainly for chlortetracyclin (fig. 2a)

4. Protein level in feed has no consistent effect on antibiotic stability.

5. Pelleting process increases losses, mostly for spiramycin ( $3 \circ \mathrm{p}$. 100 ) in non fat supplemented feeds (fig. $5 c$ ). On the other hand, fat seems to exert a protective effect against degradation due to pelleting (fig. $5 a$ ).

6. Influence of level of antibiotic supplementation is rather small, except for oxytetracyclin.

\section{RÉFÉRENCES BIBLIOGRAPHIQUES}

Al.bert A., 1953. Avidity of terramycin and aureomycin for metallic cations. Valure, 172, 201 .

Analytical Methods Committef, ig53. The determination of penicilline, chlortetracycline and oxytetracycline in diet supplements and compound feedstuffs. Analyst., 88, $835^{-8} 5^{\circ}$.

Barbiers A. R., NFFF J., 1966. Development of an assay for neomycin in feeds. J. Ass. off. Agric. Chem., 49, $125^{6}$.

Binkley S. 13., 1955. Biochemistry of antibiotics. Ann. Rev. Biochem., 24, 597-627.

Diding N. A., 1954. Stability of tetracyclines. Svensk. Farm. Tidskr., 58, 745-75I.

Esposito R. G., 1952. Stability of various penicillin salts used for growth promotion in animal feeds. Fedn. Proceed., 11, 208.

Fox P. F., I965. Destruction of the properties of some antibiotics by hyclrogen peroxide. J. Dairy Sci., 43, 1 II 6 -III8.

Geiger W. B., I946. Inactivation of streptomycin and its practical application. Proc. Soc. Exp. Biol. Med., 61, I87-192.

Goldberg II. S., 1959. Antibiolics, their chemistry and non medical uses. Van Nostrand, New York.

Grove D. C., Randal. W. A., I955. Assay methods of antibiotics. Medical Encyclopedia inc., New York.

HARMS R. If., WiLSON H. R., HALdroup P. W., ig64. The effects of certain estrogenic hormones, terephtalic acid and calcium level upon oxytetracyclin utilization. Poull. Sci., 43, 970-973.

Hollenbeck C. M., 1954. Stability of procain penicillin in feeds. Poult. Sci., 33, 425-427.

HusaAs 0., 1967. An antibiotic. Proceed. Holmenkollen Syntpos., Oslo, 75-84.

Jounandet C., Aumaitre A., Salmon-Legagneur E., I 964 . Influence des antibiotiques sur la croissance des porcelets sevrés à 5 semaines. Ann. Zoolech., 13, h.-s. I, I I 3 - I 27.

Katz S. E., 1963. Comparison of chemical and microbiological methods for the détermination of procaine penicillin in premixes and mixed feeds. I. Ass. off. Agric. Chem., 46, 429-433.

Katz S. E., Fassbender C. A., I 967 . Studies in the stability of chlortetracyclin in mixed feeds. Epimerization of chlortetracyclin. J. Ass. off. Agric. Chem., 50, $821-827$.

Kawabata T., SakaguciII G., Nakamura Y., Akano 'T'., 1960. Factors affecting the decomposition of oxytetracycline and chlortetracycline. J. Antibiot. Tokyo, 13, 180-183.

Koroleva V. P., Penova I. I., I 964 . Stabilité des préparations de tétracycline et de pénicilline dans les aliments mélangés (en russe). Trud. Inst. eksp. Vet. Mosk., 230, 329-334.

Laboratoire Rhone-Poulenc, ig6r. Mélhode de dosage de la spiramycine. (Note technique).

Muller Z., Ruzicka B., Roejl J., Mattusova D., 1958. Amélioration de la stabilité de la pénicilline dans les aliments. Zivoc. Vyroba, 31, 723-752.

Price K., Zolli Z., Atkinson J., Cuther II. G,, 1957. Antibiotics inhibition. II. Studies on the inhibitory action of selected divalent cations for oxytetracycline. Anlibiotics Chemsther., 7, 689-698.

Price K., Zolli Z., Atkinson J., Luther H., i 957 . Antibiotics inhibitions. I. The effect of certain milk constituants. Antibiotics Chemother., 7, $672-688$.

Stockstad E. L., 1952. Stability of antibiotics in poultry feeds during pelleting and storage. Poull. Sci., 31, $937-939$.

Waksuan S. A., 1950. Antimicrobial properties of neomycin. J. Lab. Clin. Med., 36, 93-99.

WeinBerG; E. D., 1957. The mutual effects of antimicrobial compounds and metallic cations. Bac. Rei., 21, $46-68$.

WELCH II., I950. Comparative studies on terramycin and aureomycin : antibacterial spectrum, serum concentrations and urinary excretion. J. Amer. Pharm. Ass. Sci., 39, 185-192.

Weinstein L., 1958. Streptomycin and dehydrostreptomycin. Medical Encyclopedia, New York.

Whittet T. D., r959. Decomposition of medicaments due to excipients and containers and its prevention. Pharm. Acta Heluel., 34, 489-520.

Wornick R. G., I959. Feed pelleting and its effects on microingredients. 10th Ann. Feed. Production School, Missouri.

Wornick R. C., RUhn G. O., I962. Stability of several oleandomycin derivatives in livestock and poultry feeds products. J. Agr. Food. Chem., 10, 286-290.

Wornick R. C., I967. Antibiotic stability, a review. Proceed. 15th Research conf. Pfizer, 54-90. 